\title{
The relationship between event-related potentials, stress perception and personality type in patients with multiple sclerosis without cognitive impairment: A pilot study
}

\author{
Marta Waliszewska-Prosół ${ }^{1, A-D}$, Marta Nowakowska-Kotas ${ }^{1, A-D}$, Roman Kotas ${ }^{2, B, D}$, \\ Tomasz Bańkowski ${ }^{3, C}$, Anna Pokryszko-Dragan ${ }^{1, A, C, E, F}$, Ryszard Podemski ${ }^{1, E, F}$ \\ ${ }^{1}$ Department of Neurology, Wroclaw Medical University, Poland \\ 2 Department of Psychiatry, Regional Specialist Hospital, Legnica, Poland \\ ${ }^{3}$ Department of Cardiology, Wroclaw Medical University, Poland \\ A - research concept and design; $\mathrm{B}$ - collection and/or assembly of data; $\mathrm{C}$ - data analysis and interpretation; \\ $\mathrm{D}$ - writing the article; $\mathrm{E}$ - critical revision of the article; $\mathrm{F}$ - final approval of the article
}

Address for correspondence

Marta Waliszewska-Prosół

E-mail: marta.waliszewska@gmail.com

Funding sources

None declared

Conflict of interest

None declared

Received on September 4, 2016

Reviewed on January 2, 2017

Accepted on February 13, 2017

\begin{abstract}
Background. The clinical course of multiple sclerosis (MS) can vary significantly among patients and is affected by exogenous and endogenous factors. Among these, stress and personality type have been gaining more attention.

Objectives. The aim of this study was to investigate the parameters of event-related potentials (ERPs) with regards to stress perception and personality type, as well as cognitive performance in MS patients.

Material and methods. The study group consisted of $30 \mathrm{MS}$ patients and 26 healthy controls. Auditory ERPs were performed in both groups, including an analysis of P300 and N200 response parameters. The Perceived Stress Scale (PSS) was used in the MS group to measure the perception of stress. The D-type Scale (DS14) scale was used to determine the features of Type D personality, characterized by social inhibition and negative affectivity.

Results. The score on the PSS corresponded with a moderate or high level of stress perception in 63\% of MS patients, while $23 \%$ of patients presented with a Type D personality. P300 latencies were significantly longer $(p=0.001)$, N200 amplitudes were significantly higher $(p=0.004)$, and N200 latencies were longer in MS patients than in the controls. Strong positive correlations were found between N200 and P300 amplitudes, as well as between the DS14 and PSS results.

Conclusions. Most MS patients experience moderate to severe stress. ERP abnormalities were found in MS patients who did not have overt cognitive impairment and showed correlations with stress levels and negative affectivity. Event-related potentials may be useful in assessing the influence of stress and emotions on the course of MS.
\end{abstract}

Key words: multiple sclerosis, event-related potentials, stress perception, personality type

DOI

$10.17219 /$ acem/68944

Copyright

Copyright by Author(s)

This is an article distributed under the terms of the

Creative Commons Attribution Non-Commercial License

(http://creativecommons.org/licenses/by-nc-nd/4.0/) 


\section{Introduction}

Multiple sclerosis (MS) is a central nervous system inflammatory/autoimmune disorder. Multifocal demyelinating lesions are accompanied by axonal loss due to neurodegenerative processes. ${ }^{1}$ The clinical course of MS can vary significantly among patients. This renders it less predictable, especially at the onset of the disease. Among other factors, stress and personality type have recently been increasingly associated with the individual course of MS. A relationship between the course of MS and stress has been found. Stress can aggravate MS symptoms, while disability and the chronicity of the disease result in stress, creating a vicious circle. However, studies in this field are inconclusive and the value of the tests used to measure the intensity of stress is constantly disputed. ${ }^{2-4}$

Event-related potentials (ERPs) are an electrophysiological method considered to reflect a subject's cognitive and emotional reaction to a mental task. Parameters of ERPs, particularly the most commonly analyzed P300 component, are mainly used in combination with neuropsychological tools as a measure of cognitive impairment in the course of various central nervous system (CNS) diseases, including MS. Those parameters may be affected by stress and anxiety. ${ }^{5}$ Therefore, it seems interesting to analyze ERPs in combination with the patient's susceptibility to stress and the intensity of stress itself. Such analysis could prove the usefulness of the method as a biomarker in this field.

The aim of our pilot study was to analyze event-related potential parameters in MS patients, with regard to the perceived stress level and personality features.

\section{Material and methods}

The study was carried out on 30 patients with relapsingremitting MS (26 women and 4 men; mean age: 34.9 years) and 26 healthy controls, matched to the patients with regard to age, gender and education level (22 women and 4 men; mean age: 34.6 years). The patients enrolled in the study were diagnosed with clinically definite MS by an experienced neurologist according to the McDonald criteria. ${ }^{6}$ All patients underwent at least 1 magnetic resonance imaging (MRI) examination, which revealed dissemination of lesions in both space and time. The duration of the disease varied from 1 to 18 years (mean: 6.2 years; standard deviation (SD): 4.3) and the mean Expanded Disability Status Scale (EDSS) score was 1.8 (range: 1-3.5; median score: 1.5$){ }^{7}$ All the patients were in the course of treatment with disease-modifying agents, including interferon beta and glatiramer acetate. Exclusion criteria included severe cognitive impairment (patients unable to follow the test instructions), mood disorders (such as depression), progressive forms of MS, concomitance of other neurological or systemic disorders that may have influenced the cognitive performance and/or ERP results, severely decreased visual acuity, and hearing loss.

The study was approved by the Commission of Bioethics at the Wroclaw Medical University. All the subjects provided informed consent prior to their inclusion in the study.

A battery of tests was performed in the MS group to measure the perception of stress, the presence of Type D personality, and the basic level of cognitive performance (Symbol Digit Modalities Test - SDMT). ${ }^{8,9}$

The Perceived Stress Scale (PSS) was developed to measure the degree to which a recent situation in one's life is perceived as stressful - the higher the score (range $0-40)$, the higher the perception of stress. ${ }^{10}$ In this study, Polish 10-point version of the scale was used. ${ }^{11}$ In addition, sten scores were calculated; they indicated an individual's approximate score with respect to the whole population (sten scores of 1-4 corresponded with a low perception of stress, sten scores of 5-6 corresponded with moderate stress perception and sten scores of 7-10 corresponded with a high level of perceived stress). ${ }^{11}$

The D-type Scale (DS14) is a questionnaire used to determine the features of the so-called Type D personality, believed to be associated with a higher risk of morbidity and a higher susceptibility to stress. In order to be classified as Type D, one has to score more than 10 out of 14 points in the 2 dimensions of the scale: negative affectivity and social inhibition. ${ }^{12}$

The Symbol Digit Modalities Test (SDMT) is regarded as a reliable screening test for cognitive performance (including attention and executive functions) of MS patients. ${ }^{13}$ In our study, it was performed in order to exclude patients with overt cognitive dysfunction, as well as to comparatively analyze the relationships between ERP parameters and stress, personality type and cognitive performance.

Event-related potentials were obtained from the MS patients and the controls. The multimodal ERPs were recorded in the Evoked Potential laboratory of the Department of Neurology of the Wroclaw Medical University. ERPs were induced after auditory stimulation with tones that differed in frequency (1 kHZ and $2 \mathrm{kHz}$ ) but had the same duration (200 ms) and intensity $(70 \mathrm{~dB})$. These tones were delivered binaurally through headphones. The oddball paradigm was used, where the eliciting stimuli were randomly presented in between neutral stimuli. The target stimuli occurred less frequently and constituted $20 \%$ of all stimuli, while the neutral stimuli made up $80 \%$ of each series. The recording $\mathrm{Ag} / \mathrm{AgCl}$ electrodes were placed in the sagittal plane on the head in the frontal $(\mathrm{Fz})$, central $(\mathrm{Cz})$, and parietal $(\mathrm{Pz})$ regions according to the $10-20$ system of electrode placement. Two earlobe electrodes were used as reference electrodes, and the ground electrode was placed on the forearm. Electrode impedance was controlled and kept below $5000 \Omega$. The patient was recumbent with his/hers eyes closed. The series consisting of 30 to 40 target stimuli was presented to the patient at 2 time-points, and the patient was asked to count the stimuli. The response within the filter bandpass target (between 0.3 and $70 \mathrm{~Hz}$, for $1000 \mathrm{~ms}$ ) was averaged separately for target stimuli and neutral stimuli. 
The N200 and P300 components were distinguished and their latencies and peak-to-peak amplitudes were determined.

The procedure used to record the ERPs was compliant with the International Federation of Clinical Neurophysiology (IFCN) and the standards recommended by the American Society of Electroencephalography. ${ }^{14}$ The same ERP protocol was used in the earlier studies on MS patients conducted at our site. ${ }^{5}$

The ERP parameters obtained from MS patients and controls were compared, and the relationship between ERP and PSS, personality type and the SDMT was analyzed.

\section{Statistical analysis}

The statistical analysis was performed using of STATISTICA v. 10 software (StatSoft Inc., Tulsa, USA). The normality of distribution was verified with the Shapiro-Wilk test. If a normal distribution was stated, the groups were compared using the parametric Student's t-test. If the parameter value distributions differed significantly from a normal distribution, the non-parametric Mann-Whitney U test was used to compare the groups. The ANOVA test was used to compare more than 2 variables in the non-combined groups. Correlation coefficients were calculated and assessed using a standard Pearson coefficient. To assess relationships between 2 variables, $\mathrm{p} \leq 0.05$ was considered statistically significant and p-value of 0.02-0.05 indicated a p-trend.

\section{Results}

\section{Symbol Digit Modalities Test results}

A SDMT was performed in all the patients, and a mean result of $55.6 \pm 7.94$ points was obtained. None of the patients obtained scores lower than those corresponding to normal for the age range. The result of the SDMT did not correlate significantly with P300 or N200 latencies and amplitudes. A negative correlation $(\mathrm{R}=-0.41 ; \mathrm{p}<0.05)$ was found between the SDMT and the negative affectivity subscale of the DS14. No other correlations were found between the SDMT and the remaining test results.

\section{Perceived Stress Scale and D-type Scale personality test results}

The perceived level of stress was measured using the PSS. The mean score was 16.6 points (range: $5-26$ points). The level of stress was determined as low in 11 individuals (37\%), moderate in 6 individuals (20\%) and high in 13 individuals (43\%) when expressed in sten scores. A high level of stress was observed in $50 \%$ of men and $42 \%$ of women. A moderate level of stress was found in $0 \%$ of men and $23 \%$ of women, and low stress levels were seen in $50 \%$ of men and $34 \%$ of women. A Type D personality was found in 7 individuals (23\% of the total study group), all of whom were women.

\section{P300/N200 between groups}

The 2 components of the ERP - N200 and P300 - were performed in all the subjects. The mean N200 amplitude values and $\mathrm{P} 300$ latency values registered in all 3 recording sites $(\mathrm{Fz}, \mathrm{Cz}$ and $\mathrm{Pz})$ were significantly higher in the group of MS patients than in the controls. A trend was also noted in the group of patients with MS toward longer latencies of the N200 recorded in the Fz and Pz sites ( $\mathrm{p}=0.17$ and $\mathrm{p}=0.18$, respectively) than in the control group (Table 1).

Table 1. The mean value of the latency [ms] and amplitude [uV] of N200 and P300 potentials in patients with MS and in the control group

\begin{tabular}{|c|c|c|c|c|c|}
\hline \multirow{2}{*}{ ERP } & \multicolumn{2}{|c|}{ Patients with MS $(n=30)$} & \multicolumn{2}{|c|}{ Control group $(n=26)$} & \multirow{2}{*}{$p$-value } \\
\hline & mean & SD & mean & SD & \\
\hline \multicolumn{6}{|c|}{ Latency [ms] } \\
\hline $\mathrm{N} 200 \mathrm{Fz}$ & 211.7 & 21.6 & 207.0 & 21.1 & 0.17 \\
\hline $\mathrm{Cz}$ & 208.8 & 20.5 & 205.3 & 20.7 & 0.53 \\
\hline$P z$ & 214.2 & 26.1 & 205.8 & 19.0 & 0.18 \\
\hline P300 Fz & 338.4 & 23.5 & 314.5 & 20.3 & 0.0002 \\
\hline $\mathrm{Cz}$ & 336.4 & 23.9 & 315.6 & 22.3 & 0.001 \\
\hline $\mathrm{Pz}$ & 338.6 & 21.4 & 318.3 & 23.4 & 0.001 \\
\hline \multicolumn{6}{|c|}{ Amplitude [uV] } \\
\hline N200 Fz & 7.0 & 3.5 & 4.5 & 3.9 & 0.006 \\
\hline $\mathrm{Cz}$ & 6.7 & 4.0 & 3.9 & 3.6 & 0.001 \\
\hline $\mathrm{Pz}$ & 5.9 & 5.4 & 2.9 & 2.3 & 0.004 \\
\hline P300 Fz & 8.9 & 6.4 & 8.6 & 8.0 & 0.7 \\
\hline $\mathrm{Cz}$ & 8.7 & 5.7 & 8.4 & 8.0 & 0.8 \\
\hline $\mathrm{Pz}$ & 8.4 & 7.1 & 8.4 & 6.9 & 0.59 \\
\hline
\end{tabular}

ERP - event-related potential; MS - multiple sclerosis; SD - standard deviation; Fz - frontal region; Cz - central region; Pz - parietal region. 


\section{N200 and DS14}

The presence of a Type D personality, determined by the DS14 test, did not correlate significantly with any latency or amplitude values of the N200 component. However, a trend toward an inverse correlation between a Type D personality and the latency of N200 in the Pz recording site was observed (Table 2). Similarly, no statistically significant correlations were found between the latencies of the N200 at any of the recording sites and the components of the DS14 test, i.e., negative affectivity and social inhibition. An inversely proportional correlation was found between the N200 latencies recorded at the $\mathrm{Cz}$ and Pz sites and the 2 constituents of DS14. In the case of the N200 latency recorded at the $\mathrm{Cz}$ and Pz sites, there was an inversely proportional correlation between individual components of the DS14 (Table 3). The amplitude of N200 in the $\mathrm{Fz}, \mathrm{Cz}$ and $\mathrm{Pz}$ sites strongly correlated with negative affectivity. No such tendency was found in the case of social inhibition (Table 4). When comparing patients with and without Type D personality, a trend towards longer N200 latencies was found at the $\mathrm{Cz}$ and Pz sites

Table 2. The correlation of Type D personality and parameters of the N200 wave

\begin{tabular}{|l|c|c|}
\multicolumn{1}{|c|}{ Correlation } & $\mathrm{R}^{*}$ & $\mathrm{p}$-value \\
\hline D - type \& N200 Cz lat & 0.33 & 0.07 \\
\hline D - type \& N200 Pz lat & -0.36 & 0.05 \\
\hline D - type \& N200 Fz lat & -0.20 & 0.3 \\
\hline D - type \& N200 Cz amp & -0.0033 & 0.99 \\
\hline D - type \& N200 Pz amp & 0.1137 & 0.55 \\
\hline D - type \& N200 Fz amp & 0.0570 & 0.77 \\
\hline
\end{tabular}

Fz - frontal region; $\mathrm{Cz}$ - central region; $\mathrm{Pz}$ - parietal region; amp - amplitude.

Table 3. Correlation of negative affectivity and social inhibition estimated using the DS14 scale and CERP latencies assessed using the standard Pearson coefficient

\begin{tabular}{|l|c|c|}
\multicolumn{1}{|c|}{ Correlation } & $\mathrm{R}^{*}$ & $\mathrm{p}$-value \\
\hline NA \& N200 Cz lat & -0.28 & 0.13 \\
\hline NA \&N200 Pz lat & -0.28 & 0.14 \\
\hline NA \&N200 Fz lat & -0.15 & 0.43 \\
\hline NA \& P300 Cz lat & 0.02 & 0.91 \\
\hline NA \& P300 Pz lat & -0.04 & 0.83 \\
\hline NA \& P300 Fz lat & -0.04 & 0.85 \\
\hline SI \& N200 Cz lat & -0.25 & 0.19 \\
\hline SI \&N200 Pz lat & -0.32 & 0.09 \\
\hline SI \&N200 Fz lat & -0.1 & 0.59 \\
\hline SI \& P300 Cz lat & 0.18 & 0.35 \\
\hline SI \& P300 Pz lat & 0.11 & 0.58 \\
\hline SI \& P300 Fz lat & 0.19 & 0.30 \\
\hline
\end{tabular}

$\mathrm{NA}$ - negative affectivity; SI - social inhibition; Fz - frontal region; $\mathrm{Cz}$ - central region; Pz - parietal region; lat - latency.
Table 4. Correlation of negative affectivity and social inhibition estimated using the DS14 scale and CERP amplitudes assessed using the standard Pearson coefficient

\begin{tabular}{|l|c|c|}
\multicolumn{1}{|c|}{ Correlation } & $\mathrm{R}^{*}$ & $\mathrm{p}$-value \\
\hline NA \& N200 Fz amp & 0.40 & 0.03 \\
\hline NA \& N200 Cz amp & 0.46 & 0.01 \\
\hline NA \& N200 Pz amp & 0.53 & 0.001 \\
\hline SI \& N200 Fz amp & -0.13 & 0.51 \\
\hline SI \& N200 Cz amp & -0.08 & 0.69 \\
\hline SI \& N200 Pz amp & 0.07 & 0.72 \\
\hline NA \& P300 Fz amp & & \\
\hline NA \& P300 Cz amp & -0.21 & 0.27 \\
\hline NA \& P300 Pz amp & -0.28 & 0.13 \\
\hline SI \& P300 Fz amp & -0.03 & 0.87 \\
\hline SI \& P300 Cz amp & 0.01 & 0.94 \\
\hline SI \& P300 Pz amp & -0.23 & 0.23 \\
\hline
\end{tabular}

Fz - frontal region; $\mathrm{Cz}$ - central region; $\mathrm{Pz}$ - parietal region; NA - negative affectivity; SI - social inhibition; amp - amplitude.

recorded in the group of patients without Type D features $(212.5 \pm 16.5$ vs $196.7 \pm 28.3, \mathrm{p}=0.07$ and $219.3 \pm 23.7$ vs $197.6 \pm 28.4, \mathrm{p}=0.05$, respectively).

\section{P300 and DS14}

Type D personality did not correlate with any parameter of the P300 wave (Table 5). No correlation was found between the latency and amplitude values of P300 and the components of the DS14 scale at any of the recording sites (Table 3). An inverse correlation was observed between the amplitude of $\mathrm{P} 300$ at the $\mathrm{Cz}$ site and negative affectivity $(\mathrm{R}=-0.28 ; \mathrm{p}=0.13)$ (Table 4$)$.

Table 5. Correlation of Type D personality and parameters of the P300 wave

\begin{tabular}{|l|c|c|}
\hline \multicolumn{1}{|c|}{ Correlation } & $\mathrm{R}^{*}$ & $\mathrm{P}$-value \\
\hline D - type \& P300 Cz lat & 0.1237 & 0.52 \\
\hline D - type \& P300 Pz lat & 0.0920 & 0.63 \\
\hline D - type \& P300 Fz lat & 0.0716 & 0.71 \\
\hline D - type \& P300 Cz amp & -0.2074 & 0.27 \\
\hline D - type \& P300 Pz amp & -0.0981 & 0.61 \\
\hline D - type \& P300 Fz amp & -0.1409 & 0.49 \\
\hline
\end{tabular}

ERP - event-related potential; MS - multiple sclerosis; SD - standard deviation; Fz - frontal region; Cz - central region; Pz - parietal region; lat latency; amp - amplitude.

\section{N200/P300 and PSS}

A statistically significant directly proportional relationship was found between the amplitudes of the N200 component at each recording site and the 10-point PSS and 10sten PSS (Table 6). No statistically significant differences 
Table 6. Correlations between the latency and amplitude of N200 and PSS10 points and sten scores assessed using the standard Pearson coefficient

\begin{tabular}{|l|c|c|}
$\begin{array}{c}\text { N200 } \\
\text { amplitude } \\
\text { vs PSS 10 }\end{array}$ & $R$ & p-value \\
\hline Fz & 0.37 & 0.04 \\
\hline Cz & 0.43 & 0.02 \\
\hline Pz & 0.45 & 0.01 \\
\hline \multicolumn{2}{|c|}{ N200 amplitude vs PSS stens } \\
\hline Fz & 0.38 & 0.04 \\
\hline Cz & 0.42 & 0.02 \\
\hline Pz & 0.48 & 0.01 \\
\hline \multicolumn{2}{|c|}{ N200 latency vs PSS 10 } \\
\hline Cz lat & -0.21 & 0.26 \\
\hline Pz lat & -0.18 & 0.35 \\
\hline Fz lat & -0.08 & 0.69 \\
\hline & N200 latency vs PSS stens \\
\hline Cz & -0.23 & 0.21 \\
\hline Pz & -0.21 & 0.27 \\
\hline Fz & -0.07 & 0.7 \\
\hline
\end{tabular}

PSS 10 - the perceived stress scale; Fz - frontal region; Cz - central region; $\mathrm{Pz}$ - parietal region.

were found between the latency of N200, the amplitude and the latency of P300 and the PSS10 (expressed in points or stens). An inversely proportional trend was observed between the P300 amplitudes recorded at Cz, 10-sten PSS $(\mathrm{R}=-0.25 ; \mathrm{p}=0.18)$ and the latency of the P300 potential recorded at $\mathrm{Cz}$ and 10 -sten PSS $(\mathrm{R}=-0.24 ; \mathrm{p}=0.2)$.

Based on the PSS test results, the patients were divided into 3 groups depending on the intensity of perceived stress (Group I: $1-4$ stens, Group II: 5-6 stens and Group
III: 7-10 stens). The ERP parameters were assessed in all groups. Patients with high levels of perceived stress had significantly larger N200 amplitudes at the Pz recording site than those with low levels of perceived stress $(8.2 \pm 4.4$ vs $3.9 \pm 3.3 ; \mathrm{p}=0.03$ ). A trend was also found toward higher N200 amplitudes at the $\mathrm{Cz}$ recording site $(\mathrm{p}=0.06)$. The remaining ERP results did not show any statistically significant differences with regard to PSS results (Table 7).

\section{Discussion}

The present study investigated the level of perceived stress and personality type, as well as their correlation with electrophysiological parameters, in MS patients.

More than $60 \%$ of the MS patients experienced moderate or severe stress. Our data is comparable with the findings of Senders et al. (16.6 points vs 16.55 points), but is significantly lower than the results of Artemiadis et al. (16.6 points vs 24.9 points) and Pritchard et al. (16.6 points vs 21.22 points). ${ }^{15-17}$ Those differences can be attributed to the larger sample size in our study and to the characteristics of our study group (a relatively low level of disability and short duration of illness, or the use of appropriate immunomodulatory treatment). Type $\mathrm{D}$ personality occurred much less commonly in the study group (23\%) than in the general Polish population (34.8\%). It was comparable to healthy populations in other European countries (16.6-38.5\%). ${ }^{12,18-21}$ Our results were similar to those obtained in patients with diabetes (20-29\%), but lower than those obtained in patients with cardiovascular disease (31-72.1\%) or on dialysis (41\%). ${ }^{18,22}$ In the study by Dubayova et al., Type D personality occurred much more commonly in MS patients (44.5\%) than in our study. ${ }^{23}$ However, that study was carried out on patients with a higher disability level (mean EDSS: 3.0 )

Table 7. The mean values of the latency [ms] and amplitude [uV] of N200 and P300 potentials in patients with MS divided into 3 groups according to the score obtained in PSS

\begin{tabular}{|c|c|c|c|c|c|c|c|}
\hline \multirow[t]{2}{*}{ ERP } & \multicolumn{2}{|c|}{$\begin{array}{c}\text { Group } 1 \text { (low) } \\
n=11\end{array}$} & \multicolumn{2}{|c|}{$\begin{array}{c}\text { Group } 2 \text { (medium) } \\
n=6\end{array}$} & \multicolumn{2}{|c|}{$\begin{array}{c}\text { Group } 3 \text { (high) } \\
n=13\end{array}$} & \multirow[t]{2}{*}{$p$-value } \\
\hline & mean & $\mathrm{SD}$ & mean & $\mathrm{SD}$ & mean & SD & \\
\hline N200 Fz lat & 214.1 & 26.0 & 215.2 & 8.8 & 208.0 & 22.4 & 0.68 \\
\hline N200 Fz amp & 5.9 & 4.0 & 5.9 & 3.2 & 8.4 & 2.7 & 0.19 \\
\hline N200 Cz lat & 212.5 & 20.6 & 210.7 & 24.6 & 204.8 & 19.4 & 0.39 \\
\hline N200 Cz amp & 5.0 & 3.1 & 6.2 & 4.7 & 8.4 & 4.0 & 0.06 \\
\hline N200 Pz lat & 216.1 & 21.1 & 213.7 & 25.1 & 212.9 & 31.7 & 0.64 \\
\hline N200 Pz amp & 3.9 & 3.3 & 4.4 & 2.7 & 8.2 & 4.4 & 0.03 \\
\hline P300 Fz lat & 337.5 & 21.5 & 347.0 & 18.7 & 335.3 & 27.4 & 0.36 \\
\hline P300 Fz amp & 9.7 & 7.6 & 11.0 & 6.7 & 7.2 & 5.0 & 0.45 \\
\hline P300 Cz lat & 334.5 & 29.3 & 347.7 & 18.1 & 332.8 & 21.3 & 0.25 \\
\hline P300 Cz amp & 10.1 & 6.6 & 10.5 & 6.2 & 6.7 & 4.3 & 0.38 \\
\hline P300 Pz lat & 338.8 & 23.3 & 348.5 & 19.5 & 333.9 & 20.5 & 0.27 \\
\hline P300 Pz amp & 8.3 & 8.8 & 12.6 & 8.3 & 6.4 & 3.9 & 0.25 \\
\hline
\end{tabular}

ERP - event-related potential; MS - multiple sclerosis; SD - standard deviation; Fz - frontal region; Cz - central region; Pz - parietal region; lat - latency; amp - amplitude. 
and suffering from various types of the disease (70\% relapsing-remitting, 30\% secondary or primary progressive).

ERPs are formed in extensive neuronal CNS networks. The P300 component, which is the most frequently analyzed parameter in clinical practice, arises in the frontal lobe, medial temporal lobe, cingulate gyrus, thalamus, and structures of the limbic system. ${ }^{24,25}$ The P300 wave reflects the complex neuronal processes involved in the reception and processing of sensory information and selective attention, indicating decision making and memory updating processes. The parameters of the P300 potential are characterized by large variability among individuals and can be influenced by numerous endogenous and exogenous factors (age, personality, intelligence, hemispheric dominance, fatigue, physical activity, drugs, smoking, and alcohol consumption). ${ }^{26}$

The latency of the P300 wave corresponds to the time needed for an impulse to be recognized and classified, and is a marker of the rate of information processing. The P300 amplitude (maximal near the centro-parietal site) corresponds to short-term memory, attention and involvement of the subject in the task. ${ }^{26}$

The N200 ERP component is analyzed much less frequently. It is generated in the sensory and frontal areas and is thought to be associated with the initial (subconscious) identification of a stimulus and the activation required to complete the given task.

In our study, we found MS patients to have longer P300 latencies than healthy controls. A trend towards a longer N200 latency was obtained at both the Fz and Pz sites.

In the available literature on MS, a prolongation of the P300 latency was most frequently described. Such prolongation correlated with the severity of white matter damage in the course of demyelination, the severity of cognitive impairment and the rate of depression. ${ }^{27,28}$ Some authors also found a prolongation of the N200 latency. ${ }^{29-31}$ Relationships between ERP parameters and the duration of the disease or the degree of disability were more disputable. ${ }^{27,28,32}$ Sundgren et al. pointed to the prognostic importance of the P300 parameters. ${ }^{33}$ They noted that the progression of cognitive disorders was accompanied by an increased latency and decreased amplitude of the P300 wave. This progression was slower in patients with an abnormality of only 1 parameter.

All the patients in our study had a normal SDMT result; thus, according to the screening test, they did not have significant cognitive impairment. It is interesting that there was no correlation between the SDMT result and the ERP parameters. This may suggest that ERP may be used to disclose subtle cognitive deficits.

We did not analyze the relationships between ERP parameters and other disease-related variables (disease duration or EDSS). However, all our patients presented with only mild disability (mean EDSS: 1.8). Auditory modality of ERP was also chosen to further eliminate the impact of MSrelated neurological deficit upon electrophysiological parameters (with the auditory pathway much less commonly affected in the course of MS than the visual one).

We found that the patients with MS in our study had higher N200 amplitudes than the control group. Similar findings were not found in the literature. The higher N200 amplitudes in our patients may suggest increased cortical and subcortical activity. This may be explained by compensatory mobilization of larger neuronal networks (due to a decline in neuronal function) in order to ensure better stimulus analysis, or perhaps a more intensive neuronal stimulation caused by higher stress levels. A long-term exposure to stress has been shown to lead to a dysfunction of the bioelectric brain activity. This may particularly occur in the course of demyelinating inflammatory diseases, hormonal activation, activation of proinflammatory cytokines, oxidative stress, or excitotoxicity. ${ }^{34-36}$

Our findings seem to support the association between N200 amplitude and stress. We found higher N200 amplitudes in MS patients with a higher level of perceived stress and a significant correlation between the amplitude of the N200 wave and negative affectivity in the DS14 scale. No such correlation was found in the case of social inhibition. The influence of stress on the initial stages of stimulus processing (corresponding with N200 parameters) may be explained by the subjects' perception that an associated mental task is difficult. Senkowski and Herrmann conducted ERP in healthy subjects using a visual discrimination task, with either an easy or difficult version. ${ }^{37}$ The authors showed that the N200 amplitude was higher when subjects carried out the more difficult version of the task. They attributed this phenomenon to early hyperactivity in the cerebral cortex in preparation for a difficult task. ${ }^{38}$

Electrophysiological parameters such as contingent negative variation (CNV) and ERP parameters (mainly amplitude) may be modulated by stress, anxiety and personality in patients and healthy subjects. CNVs were larger in subjects with high perceived stress and high levels of arousal. Similar correlations were found in subjects with prolonged stress, which was measured using the PSS. An interesting finding was made in subjects with high anxiety levels: they were able to perform tasks at a similar level to subjects with much lower anxiety levels. The former subjects had higher CNV amplitudes, which was explained by the need for more processing resources in order to maintain adequate performance. Anxiety and the perception of a stimulus considered threatening may cause more involvement of neuronal networks. On the other hand, an individual's engaging of greater cognitive reserves in preliminarily analyzing a stimulus may lead to depletion of those reserves, causing fatigue and emotional tension (another vicious circle?). ${ }^{39-41}$ Our findings of increased N200 amplitudes in MS patients with high perceived stress and in patients with negative affectivity support the above described phenomenon.

This study focused on the topic of the relationships between perceived stress, personality traits and 
neuroelectrophysiological parameters, which have remained poorly understood. The results suggest that evaluating these aspects in patients with MS of short disease duration and a low degree of disability may help to identify those who require more psychological support. Event-related potentials seem to deserve attention as an electrophysiological indicator of susceptibility to stress that may be used alongside psychological tests.

The limitations of our study include the small size of the study group and using only 1 time-point in a study of patients with MS, which is known to have a fluctuating course. However, ours was a pilot study whose findings might encourage further research. Our future studies will focus on monitoring stress levels and ERP parameters in the course of the disease and on analyzing the relationship between stress, personality type and the course of the disease (natural or modified using immunomodulatory treatment).

\section{Conclusions}

The majority of MS patients experience moderate or severe stress, which needs to be addressed with appropriate psychological support. Abnormalities of ERP were found in the MS patients without any overt cognitive decline and showed correlations with measures of stress and negative affectivity (one of the dimensions of the D-type personality scale). Event-related potentials may be considered in the assessment of the influence of stress and emotions on the course of MS.

\section{References}

1. Selmaj K. Stwardnienie rozsiane - kryteria diagnostyczne i naturalny przebieg choroby. Pol Przeg Neurol. 2005;1:99-105.

2. Wolińska A. Psychological stress and multiple sclerosis. Neuropsychiat neuropsychol. 2012;7:184-189.

3. Strober L, Arnett P. Unemployment among women with multiple sclerosis: The role of coping and perceived stress and support in the workplace. Psychol Health Med. 2015;12:1-9.

4. Mohr DC, Goodkin DE, Bacchetti P, et al. Psychological stress and the subsequent appearance of new brain MRI lesions in MS. Neurology. 2000;55:55-61.

5. Polich J. P300 clinical utility and control of variability. J Clin Neurophysiol. 1998;15:14-33.

6. Polman CH, Reingold SC, Banwell B, et al. Diagnostic criteria for multiple sclerosis: 2010 revisions to the McDonald criteria. Ann Neurol. 2011;69:292-302.

7. Kurtzke JF. Historical and clinical perspectives of the Expanded Disability Status Scale. Neuroepidemiology. 2008;31:1-9.

8. Rao S. A Manual for the Brief, Repeatable Battery of Neuropsychological tests in multiple sclerosis. New York, NY: National Multiple Sclerosis Society; 1991.

9. Smith A. The symbol-digit modalities test: A neuropsychologic test of learning and other cerebral disorders. In: Helmuth J, ed. Learning disorder. Seattle, WA: Special Child Publications; 1968:83-91.

10. Cohen S, Kamarck T, Mermelstein E. A global measure of perceived stress. J Health Soc Behav. 1983;24:385-396.

11. Juczyński Z, Ogińska-Bulik N. Narzędzia pomiaru stresu i radzenia sobie ze stresem [in Polish]. Warszawa: Pracownia Testów Psychologicznych; 2009.

12. Denollet J. DS14: Standard assessment of negative affectivity, social inhibition, and type D personality. Psychosom Med. 2005;67:89-97.
13. Parmenter BA, Weinstock-Guttman B, Garg N, Munschauer F, Benedict RH. Screening for cognitive impairment in multiple sclerosis using the Symbol Digit Modalities Test. Mult Scler J. 2007;13:52-57.

14. Duncan C, Barry R, Connolly J, et al. Event-related potentials in clinical research: Guidelines for eliciting, recording, and quantifying mismatch negativity, P300, and N400. Clin Neurophysiol. 2009;120: 1883-1908.

15. Senders A, Bourdette D, Hanes D, Yadav V, Shinto L. Perceived stress in multiple sclerosis: The potential role of mindfulness in health and wellbeing. J Evid Based Complementary Altern Med. 2014;19:104-111.

16. Artemiadis A, Vervainioti A, Alexopoulos E, Rombos A, Anagnostouli M, Darviri C. Stress management and multiple sclerosis: A randomized controlled trial. Arch Clin Neuropsychol. 2012;27:406-416.

17. Pritchard $M$, Elison-Bowers $P$, Birdsall B. Impact of integrative restoration (iRest) meditation on perceived stress levels in multiple sclerosis and cancer outpatients. Stress Health. 2010;26:233-237.

18. Ogińska-Bulik N. Type D personality in Poland: Validity and application of the Polish DS14. Pol Psychol Bul. 2009;40:130-136.

19. Williams L, O'Connor R, Howard S, et al. Type-D personality mechanisms of effect: The role of health-related behavior and social support. J Psychosom Res. 2008;64;63-69.

20. Kunst $M$, Bogaerts $S$, Winkel F. Peer and inmate aggression, Type $D$ personality and post-traumatic stress among Dutch prison workers. Stress Health. 2009;25:387-395.

21. Schiffer A, Smith O, Pedersen S, Widdershoven J, Denollet J. Type D personality and cardiac mortality in patients with chronic heart failure. Int J Cardiol. 2010;142:230-235.

22. Nefs G, Speight J, Pouwer F, Pop V, Bot M, Denollet J. Type D personality, suboptimal health behaviors and emotional distress in adults with diabetes: Results from Diabetes MILES-The Netherlands. Diabetes Res Clin Pract. 2015;108:94-105.

23. Dubayova T, Krokavcova M, Nagyova I, et al. Type D, anxiety and depression in association with quality of life in patients with Parkinson's disease and patients with multiple sclerosis. Qual Life Res. 2013;22:1353-1360.

24. Ludowig E, Bien C, Elger C. Two P300 generators in the hippocampal formation. Hippocampus. 2010;20:186-195.

25. Halgren E, Marinkovic K, Chauvel P. Generators of the late cognitive potentials in auditory and visual oddball tasks. Electroencephalogr Clin Neurophysiol. 1998;106:156-164.

26. Polich J. Meta-analysis of P300 normative aging studies. Psychophysiology. 1996;33:334-353.

27. Aminoff J, Goodin D. Long-latency cerebral event-related potentials in multiple sclerosis. J Clin Neurophysiol. 2001;18:372-377.

28. Elger T, Bethke F, Frese A, et al. Event-related potenctials in different subtypes of multiple sclerosis - A cross-sectional study. J Neurol Sci. 2002;205:35-40.

29. Pokryszko-Dragan A, Zagrajek M, Słotwiński K, Gruszka E, Bilińska M, Podemski R. Neuropsychological testing and event-related potentials in the assessment of cognitive performance in the patients with multiple sclerosis - A pilot study. Clin Neurol Neurosurg. 2009;111:503-506.

30. Kocer B, Unal T, Nazliel B, et al. Evaluating sub-clinical cognitive dysfunction and event-related potentials (P300) in clinically isolated syndrome. Neurol Sci. 2008;29:435-444.

31. Gil R, Zai L, Neau JP, et al. Event-related auditory evoked potentials and multiple sclerosis. Electroencephalogr Clin Neurophysiol. 1993;88: 182-187.

32. Pokryszko-Dragan A, Zagrajek M, Slotwinski K, et al. Event-related potentials and cognitive performance in multiple sclerosis patients with fatigue. Neurol Sci. 2016;37(9):1545-1556.

33. Sundgren M, Nikulin VV, Maurex L, Wahlin L, Piehl F, Brismar T. P300 amplitude and response speed relate to preserved cognitive function in relapsing-remitting multiple sclerosis. Clin Neurophysiol. 2015; 126:689-697.

34. Rogan M, Staubli U, LeDoux J. Fear conditioning induces associative long-term potentiation in the amygdala. Nature. 1997;390: 604-607.

35. Maes M, Mihaylova I, Bosmans E. Not in the mind of neurasthenic lazybones but in the cell nucleus: Patients with chronic fatigue syndrome have increased production of nuclear factor kappa beta. Neuro Endocrinol Lett. 2007;28:456-462. 
36. Maes M, Twisk FN, Kubera M, Ringel K. Evidence for inflammation and activation of cell-mediated immunity in myalgic encephalomyelitis/ chronic fatigue syndrome (ME/CFS): Increased interleukin-1, tumor necrosis factor-a, PMN-elastase, lysozyme and neopterin. J Affect Disord. 2012;136:933-939.

37. Senkowski D, Herrmann C. Effects of task difficulty on evoked gamma activity and ERPs in a visual discrimination task. Clin Neurophysiol. 2002;113:1742-1753.

38. Duan H, Yuan Y, Yang C, Zhang L, Zhang K, Wu J. Anticipatory processes under academic stress: An ERP study. Brain Cogn. 2015;94: 60-67.
39. Brown D, Fenwick P, Howard R. The contingent negative variation in a Go/No Go avoidance task: Relationships with personality and subjective state. Int J Psychophysiol. 1989;7:35-45.

40. Nagai $Y$, Critchley H, Featherstone E, Fenwick P, Trimble M, Dolan R. Brain activity relating to the contingent negative variation: An fMRI investigation. Neurolmage. 2004;21:1232-1241.

41. Ansari T, Derakshan N. The neural correlates of cognitive effort in anxiety: Effects on processing efficiency. Biol Psychol. 2011;86: 337-348. 